\title{
Liquen escleroso y atrófico extragenital en hombre con cáncer de próstata: ¿manifestación cutánea paraneoplásica?
}

Extragenital lichen sclerosus et atrophicus in man with prostate cancer: paraneoplastic skin manifestation?

\author{
Julián Rondón-Carvajal ${ }^{10 R C I D}$, Alejandro Castellanos-Angarita² ORCID, Laura Patricia Charry-

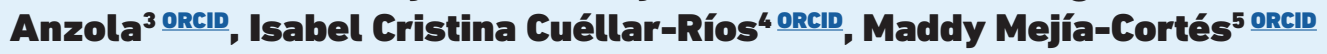

Fecha correspondencia:

Recibido: octubre 21 de 2019.

Revisado: agosto 27 de 2020.

Aceptado: octubre 20 de 2020.

Forma de citar:

Rondón-Carvajal J, Castellanos-

Angarita A, Charry-Anzola LP,

Cuéllar-Ríos IC, Mejía-Cortés

M. Liquen escleroso y atrófico

extragenital en paciente

oncológico: una asociación

infrecuente. Rev CES Med. 2020;

34(3): 221-227.

Open access

(c) Derecho de autor

Licencia creative commons

Ética de publicaciones

Revisión por pares

Gestión por Open Journal System

DOl: http://dx.doi.org/10.21615/

cesmedicina.34.3.6

ISSN 0120-8705

e-ISSN 2215-9177

Comparte

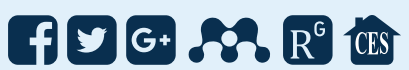

\section{Resumen}

Introducción: los síndromes esclerodermiformes se definen por la presencia de esclerosis cutánea, induración y pérdida de la elasticidad de la piel. Su diagnóstico diferencial incluye casos de escleroderma localizada, dentro de los cuales se describe el liquen escleroso y atrófico que, hasta la fecha, no se ha reconocido en el escenario de síndromes cutáneos paraneoplásicos. Resultados: se presenta el caso de un paciente con adenocarcinoma de próstata avanzado, quién desarrolló lesiones compatibles con liquen escleroso y atrófico extragenital durante el curso de su enfermedad oncológica, confirmadas por histopatología. Conclusiones: Se cuestiona la naturaleza paraneoplásica de esta entidad cutánea en este contexto clínico particular.

Palabras clave: Esclerosis; Liquen escleroso; Dermis; Cáncer.

\begin{abstract}
Introduction: Scleroderma syndromes are defined by the presence of skin sclerosis, induration and loss of skin elasticity. Their differential diagnosis includes cases of localized scleroderma, within which lichen sclerosus et atrophicus are described, which, to date, have not been recognized in the setting of paraneoplastic skin syndromes. Results: We present the case of a patient with advanced adenocarcinoma of the prostate, who developed cutaneous lesions compatible with extragenital lichen sclerosus et atrophicus during the course of the cancer, confirmed by histopathology. Conclusions: The paraneoplastic nature of this skin entity is questioned in this particular clinical context.
\end{abstract}

Keywords: Sclerosis; Lichen Sclerosus; Dermis; Cancer.

\section{Introducción}

Los síndromes esclerodermiformes son una manifestación cutánea secundaria de etiología multifactorial, dentro de las que se destacan las inmunológicas, inflamatorias, medicamentosas, metabólicas y neoplásicas (1,2). 


\section{Sobre los autores:}

1. Especialista en Medicina Interna. SURA EPS, Medellín. Grupo de investigación: Dermatosis inflamatorias.

\section{Residente de} Dermatología Pontificia Universidad Javeriana. Hospital Universitario San Ignacio. Grupo de investigación: Dermatosis inflamatorias.

\section{Especialista en} Dermatología - Magister en Epidemiología Clínica. Hospital Universitario San Ignacio. Grupo de investigación: Dermatosis inflamatorias.

\section{Especialista en} Dermatología. Hospital Universitario San Ignacio. Grupo de investigación: Dermatosis inflamatorias.

5. Especialista en Patología, subespecialista en Dermatopatología. Hospital Universitario San Ignacio. Grupo de investigación: Dermatosis inflamatorias.
Se caracterizan por la presencia de esclerosis y engrosamiento cutáneo, con presentación clínica variable (3).

La asociación entre síndromes esclerodermiformes y cáncer se ha descrito desde la década del 50 del siglo pasado, en la mayoría de casos precediendo un proceso neoplásico (4-6). Sin embargo, la escleroderma localizada -dentro de la cual se describe el liquen escleroso y atrófico- no se ha reconocido en el escenario de las manifestaciones cutáneas paraneoplásicas, aunque hay escasos descritos asociados a cáncer de mama (7). A la fecha se han reportado menos de 10 casos de síndromes esclerodermiformes relacionados con cáncer de próstata (8-10).

Presentamos el caso de un paciente con adenocarcinoma de próstata quien ,durante el curso de su enfermedad oncológica, desarrolló lesiones clínica e histológicamente compatibles con liquen escleroso y atrófico extragenital, para luego proponer su asociación como fenómeno paraneoplásico cutáneo a esta variedad de tumor sólido, según el escenario clínico particular.

\section{Caso clínico}

Un paciente de 83 años consultó al servicio de Dermatología por aparición de lesiones escleróticas en pliegue antecubital y axilas, pruriginosas, de dos años de evolución. Además, en abdomen anterior, reportaba aparición de lesiones similares, estas últimas, asintomáticas, desde hace un año.

Como antecedente de importancia fue diagnosticado tres años antes con adenocarcinoma de próstata Gleason 4+3, estadio IVB (T3N0M1b), con compromiso poliostótico, tratado con bloqueo hormonal y posterior radioterapia pélvica; además de terapia con docetaxel y denosumab ante progresión ósea, bioquímica y clínica, sin complicaciones inmediatas en el momento de la consulta.

Al examen físico, en axilas, pliegues antecubitales, región abdominal izquierda y pliegues inguinales, se encontraron placas conformadas por micropápulas, de bordes bien definidos, rugosas al tacto, pardas, con centro atrófico brillante e hipocrómico, esclerótico a la prensión (figuras 1 A y B).

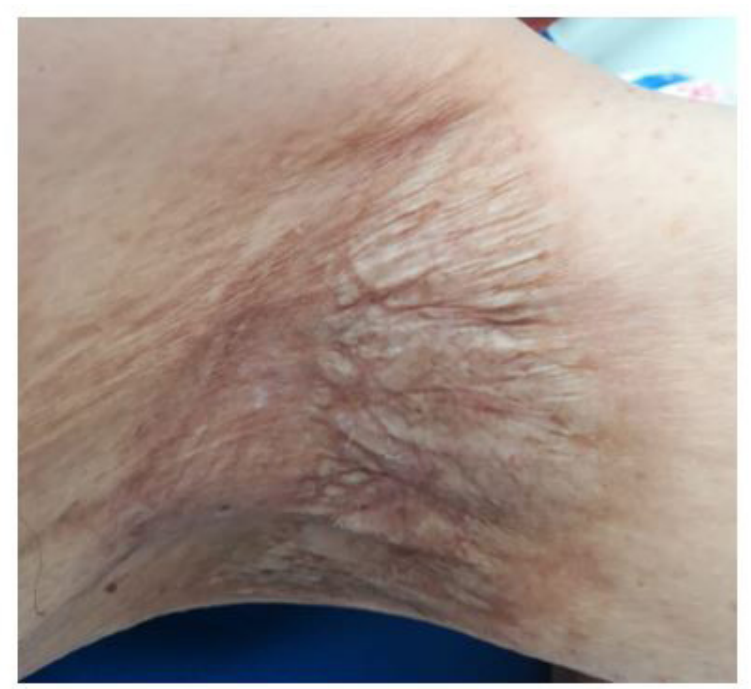

Figura 1A. Compromiso axilar por liquen escleroso y atrófico extragenital 
En axilas y pliegues inguinales se encontraron placas conformadas por micropápulas, de bordes bien definidos, rugosas al tacto, pardas, con centro atrófico brillante e hipocrómico, esclerótico a la prensión.

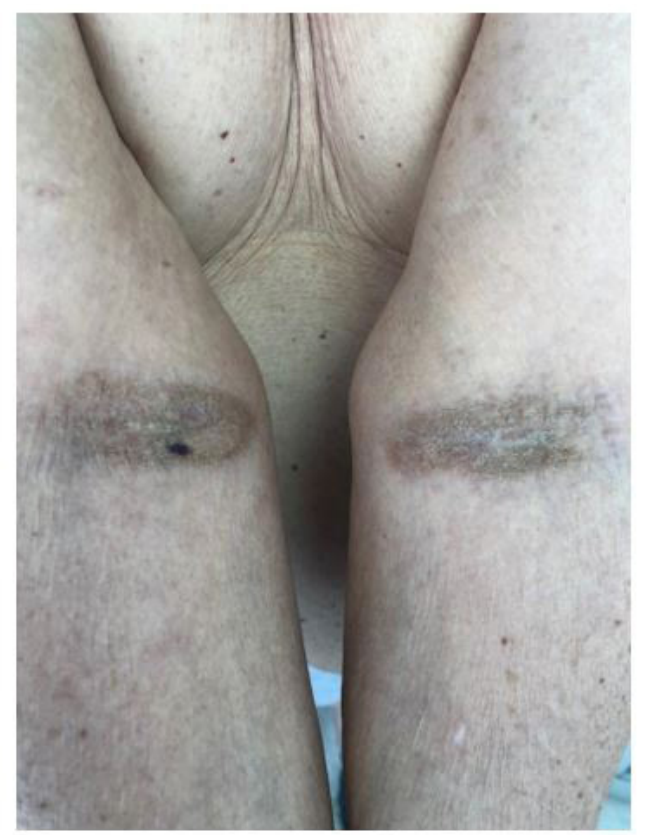

Figura 1B. Compromiso en pliegues antecubitales por liquen escleroso y atrófico extragenital

Con estos hallazgos se interrogó para un probable liquen escleroso y atrófico extragenital con criterios de severidad, dados por el número de lesiones y su progresión. Se realizó biopsia de piel de las lesiones en región periaxilar izquierda y antecubital izquierdo, confirmándose el diagnóstico (figuras 2 A y B)
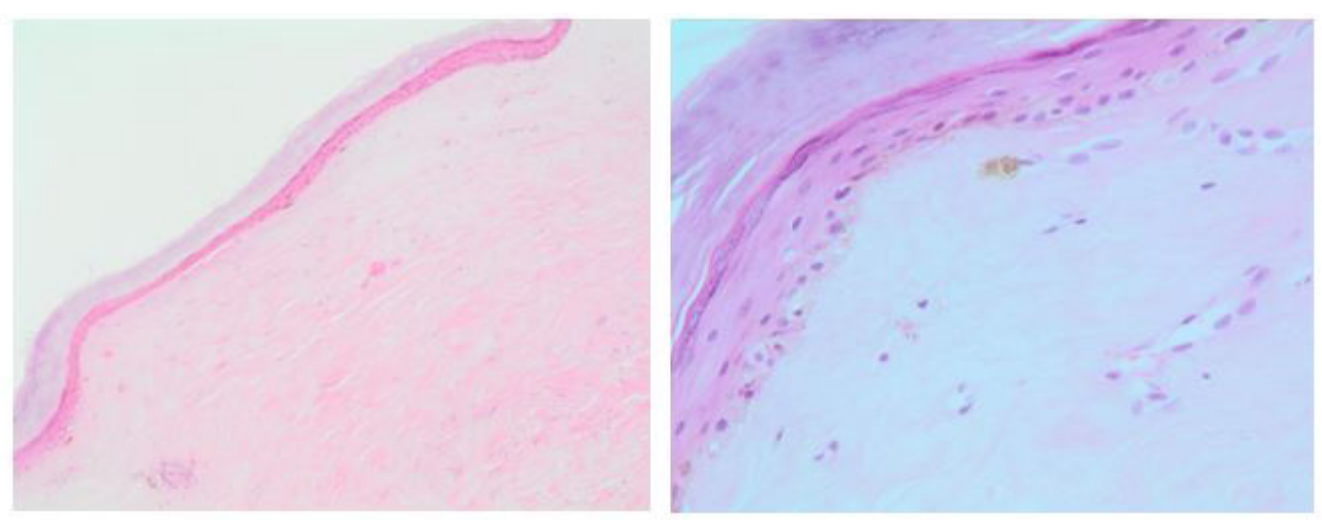

Figura 2 A y B. En A, ortoqueratosis compacta con atrofia epidérmica; en dermis, esclerosis con homogenización del colágeno. En B, daño vacuolar de la capa basal con incontinencia pigmentaria y telangiectasias en dermis superficial.

Se indicó entonces clobetasol propionato crema al 0,05 \% cada 12 horas sobre las lesiones, con posterior mejoría, continuando en seguimiento clínico y manejo oncológico para su neoplasia de base. 
El liquen escleroso y atrófico es una condición cutánea inflamatoria de evolución crónica, caracterizada por fibrosis y esclerosis, principalmente en piel genital y de predominio femenino.

\section{Discusión}

El liquen escleroso y atrófico es una condición cutánea inflamatoria de evolución crónica, caracterizada por fibrosis y esclerosis, principalmente en piel genital y de predominio femenino, descrita por primera vez en 1887 por Hallopeau (11). El compromiso extragenital aislado, como en este reporte, se ha descrito en el $2,5 \%$ de los casos en mujeres (12); sin embargo, debido a la inusual presentación en hombres, no se han realizado estudios epidemiológicos de esta variante clínica en dicha población.

Aunque esta entidad no ha sido descrito dentro de los síndromes esclerodermiformes relacionados con cáncer $(13,14)$, llama la atención la presentación atípica, es decir, en su variedad extragenital y casi sincrónica con la aparición de la neoplasia prostática, lo que podría favorecer esta asociación. A continuación, se presenta la clasificación etiológica de los trastornos esclerodermiformes más aceptada a la fecha (cuadro 1).

Cuadro 1. Espectro clínico de los trastornos fibrosantes de la piel similares a la esclerodermia

\begin{tabular}{|c|c|}
\hline Inmunomediada/inflamatoria & $\begin{array}{c}\text { Fasciitis eosinofílica } \\
\text { Enfermedad del injerto contra huésped } \\
\text { Liquen escleroso y atrófico } \\
\text { Síndrome POEMS* } \\
\text { Síndromes de sobreposición (Esclerosis sistémica/ } \\
\text { Lupus/Dermatomiositis) }\end{array}$ \\
\hline Metabólica & $\begin{array}{l}\text { Fenilcetonuria } \\
\text { Porfiria cutánea tarda } \\
\text { Hipotiroidismo (mixedema) }\end{array}$ \\
\hline Asociada a depósito & $\begin{array}{c}\text { Escleromixedema } \\
\text { Amiloidosis sistémica } \\
\text { Fibrosis sistémica nefrogénica } \\
\text { Escleredema adultorum }\end{array}$ \\
\hline Ocupacional & $\begin{array}{l}\text { Cloruro de polivinilo } \\
\text { Disolventes orgánicos } \\
\text { Silica } \\
\text { Resinas epoxídicas } \\
\text { Lipodermatoesclerosis }\end{array}$ \\
\hline Tóxica o iatrogénica & $\begin{array}{c}\text { Bleomicina } \\
\text { Pentazocina } \\
\text { Carbidopa } \\
\text { Síndrome de eosinofilia-mialgia } \\
\text { (L-triptófano) } \\
\text { Síndrome de aceite tóxico } \\
\text { (aceite de colza saturado de anilina) } \\
\text { Fibrosis post-radiación }\end{array}$ \\
\hline Genética & $\begin{array}{c}\text { Trastornos progeroides } \\
\text { (progeria, acrogeria, síndrome de Werner) } \\
\text { Síndrome de la piel rígida } \\
\text { (o distrofia facial congénita) }\end{array}$ \\
\hline
\end{tabular}

*POEMS, por sus siglas en inglés: Polineuropatía, Organomegalia, Endocrinopatía, Discrasia de células plasmáticas, Piel. Tomado de: Boin F, et al (15) 
Fisiopatológicamente, se le ha considerado como una condición autoinmune dado su asociación con otras entidades de esta índole (12); sin embargo, esto no está claramente dilucidado y también se han implicado como desencadenantes infecciones, factores hormonales, trauma, susceptibilidad genética o medicamentos (16).

El aumento en el depósito de colágeno y esclerosis en la dermis se debe a la producción de varias citocinas, como interleucina 1 (IL-1) y factor de crecimiento transformante beta (TGF-b) (16,17), compartiendo el evento fisiopatológico principal de los síndromes esclerodermiformes $(6,8)$, por lo que proponemos que, si bien el liquen escleroso no se encuentra dentro de estos síndromes, se debería considerar como una condición paraneoplásica cutánea en caso de presentarse asociado a malignidad, una vez se descarten etiologías tóxicas o asociadas a medicamentos (incluyendo algunos agentes quimioterapéuticos con potencial fibrosante, como bleomicina), siendo este el primer reporte de liquen escleroso y atrófico extragenital relacionado con adenocarcinoma prostático, descrito en la literatura.

Se han propuesto criterios diagnósticos basados en las manifestaciones clínicas e histopatológicas (18), que en el caso clínico descrito se cumplen a cabalidad (cuadro 2).

Cuadro 2. Criterios diagnósticos de liquen escleroso y atrófico*

Proponemos que, si bien el liquen escleroso no se encuentra dentro de estos síndromes, se debería considerar como una condición paraneoplásica en caso de presentarse asociado a malignidad.

\begin{tabular}{|c|c|}
\hline Criterio clínico & Placas escleróticas blancas con atrofia bien circunscrita \\
\hline Criterios histopatológicos & $\begin{array}{c}\text { Hiperqueratosis, atrofia epidérmica, degeneración por } \\
\text { licuefacción, edema intradérmico, } \\
\text { infiltración linfocítica y homogenización hialina de las fibras } \\
\text { de colágeno. }\end{array}$ \\
\hline Clasificación de severidad** & $\begin{array}{l}\text { 1. Trastorno funcional relacionado con la lesión: } 2 \text { puntos. } \\
\text { 2. Múltiples lesiones de la piel: } 1 \text { punto. } \\
\text { 3. Lesiones cutáneas que se extienden: } 1 \text { punto. }\end{array}$ \\
\hline
\end{tabular}

*El diagnóstico definitivo se establece en presencia de criterios clínicos e histopatológicos. Siempre se deben excluir la esclerodermia localizada, eccema crónico, vitiligo y liquen plano.

${ }^{* *}$ Casos de pacientes con 2 o más puntos se clasifican como severos.

Tomado de: Hasegawa M, et al (18)

El tratamiento con esteroides tópicos de alta potencia se ha descrito como la terapia de primera línea y en caso de no respuesta, los inhibidores de calcineurina, la inmunosupresión sistémica e incluso manejo quirúrgico (17-18). En los casos de síndromes esclerodermiformes paraneoplásicos se ha reportado la resolución de las manifestaciones cutáneas una vez se logra resolver o al menos controlar la progresión tumoral $(6,10)$. En este caso, el paciente obtuvo mejoría y ausencia de progresión de lesiones en piel con el manejo de primera línea; sin embargo, debido al estadio de su proceso oncológico, detener o controlar la progresión tumoral es un desafío clínico, interrogante que en esta oportunidad queda sin resolver.

\section{Conclusión}

El liquen escleroso y atrófico es una entidad que se presenta con menor frecuencia en hombres y su variedad netamente extragenital es aún más infrecuente, por lo que proponemos que debido a su debut clínico concomitante con un adenocarcinoma de próstata activo, pudiese tratarse de una nueva manifestación paraneoplásica cutánea en un paciente sin otros desencadenantes claros, incluyendo etiologías tóxicas/medicamentosas, metabólicas o incluso, exposicionales. 
Dado que esta entidad comparte un mecanismo fisiopatológico similar a los otros síndromes esclerodermiformes, se debería considerar al liquen escleroso y atrófico como una variante clínica paraneoplásica dentro de este grupo de condiciones cutáneas.
Dado que esta entidad comparte un mecanismo fisiopatológico similar a los otros síndromes esclerodermiformes, se debería considerar al liquen escleroso y atrófico como una variante clínica paraneoplásica dentro de este grupo de condiciones cutáneas.

Este caso constituye el primer reporte de una neoplasia prostática asociada a liquen escleroso y atrófico de tipo extragenital, por lo que motivamos a la comunidad médica a publicar este tipo de asociaciones, permitiendo de este modo enriquecer la evidencia científica a partir de los reportes de caso.

\section{Financiación}

No se recibió ningún tipo de financiación.

\section{Conflicto de intereses}

Ninguno por declarar por parte de los autores.

\section{Agradecimientos}

Al Hospital Universitario San Ignacio y a la Facultad de Medicina de la Pontificia Universidad Javeriana.

\section{Responsabilidades éticas}

Los autores declaran que para este artículo no se han realizado experimentos en seres humanos ni en animales. Se contó con aprobación del comité de ética del Hospital Universitario San Ignacio previo consentimiento informado del paciente.

\section{Bibliografía}

1. Ferreli C, Gasparini G, Parodi A, Cozzani E, Rongioletti F, Atzori L. Cutaneous Manifestations of Scleroderma and Scleroderma-Like Disorders: a Comprehensive Review. Clin Rev Allergy Immunol. 2017;53(3):306-336.

2. Trujillo CMC, Londoño GA, Velásquez FCJ, Restrepo MR. Reacción liquenoide secundaria al uso de adalimumab: reporte de un caso y revisión de la literatura. Med Cutan Iber Lat Am 2011;39(2):66-68.

3. Fabri M, Hunzelmann N. Differential diagnosis of scleroderma and pseudoscleroderma. J Dtsch Dermatol Ges. 2007;5(11):977-84.

4. Marek M, Rudny R. Scleroderma of geriatric age and scleroderma-like paraneoplastic syndrome - Description of two cases. Reumatologia. 2016;54(2):91-4.

5. Chung V, Moschella S, Zembowicz A, Liu V. Clinical and pathologic findings of paraneoplastic dermatoses. J Am Acad Dermatol 2006;54(5):745-762.

6. Hakkou J, Rostom S, Bahiri R, Hajjaj-Hassouni N. Paraneoplastic rheumatic syndromes: report of eight cases and review of literature. Rheumatol Int. 2012;32(6):1485-9.

7. Pappova T, Pec J, Kozarova A, Adamicova K. Cutaneous Paraneoplastic Manifestation (Morphea, Lichen Sclerosus) - Two Case Reports. Acta Medica Martiniana. 2017;17(1):28-32. 
8. Rovisco J, Serra S, Abreu P, Coutinho M, Santiago T, Inês L, et al. Paraneoplastic sclerodermiform syndrome - case reporta. Acta Reumatol Port. 2014;39(1):87-90.

9. Sharma A. Scleroderma-like Disorders. Curr Rheumatol Rev. 2018; 14(1):22-27.

10. Marasini B, Conciato L, Belloli L, Massarotti M. Systemic Sclerosis and cancer. Int J Immunopathol Pharmacol. 2009;22(3):573-8.

11. Zemmez Y, Amraoui M, Bouhamidi A, Azhari J, Ismaili N, Benzekri L, et al. Lichen scléro-atrophique extra-génital: À propos d'un cas. Pan Afr Med J. 2016;25:1-5.

12. Abbas 0, Bhawan J. Sclerosing Disorders of the Skin: An Overview with focus on histopathological features. Am J Dermatopathol 2014;10(36):763-780.

13. Deshayes S, de Boysson H, Geffray L. Les syndromes sclérodermiformes. La revue de médecine interne. 2016; 37(9): 616-624.

14. Gamarra Al, Arroyave DJ, Quintana G, Herrera FR, Matucci-Cerinic M. Historia del compromiso cutáneo de la esclerosis sistémica. Rev Colomb Reumatol. 2013; 20(3):155-70.

15. Boin F, Hummers LK. Scleroderma-like fibrosing disorders. Rheum Dis Clin North Am. 2008;34(1):199-ix.

16. Fistarol SK, Itin PH. Diagnosis and treatment of lichen sclerosus: an update. Am J Clin Dermatol. 2013;14(1):27-47

17. Pérez-López FR, Vieira-Baptista P. Lichen sclerosus in women: a review. Climacteric. 2017;20(4):339-347.

18. Hasegawa M, Ishikawa O, Asano Y, Sato S, Jinnin M, Takehara K, et al. Diagnostic criteria, severity classification and guideline of lichen sclerosus et atrophicus. J Dermatol. 2018;45(8):891-897. 